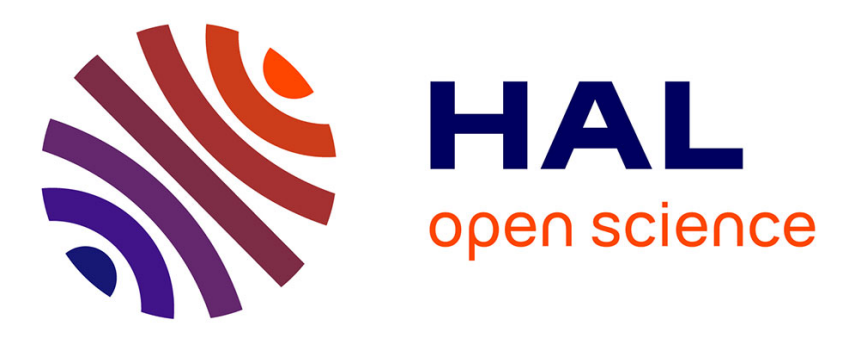

\title{
A peak detection method for identifying phase in physiological signals
}

Nicholas Mitrou, Alexandre Laurin, Taylor Dick, Jessica Inskip

\section{To cite this version:}

Nicholas Mitrou, Alexandre Laurin, Taylor Dick, Jessica Inskip. A peak detection method for identifying phase in physiological signals. Biomedical Signal Processing and Control, 2017, 31, pp.452 462. 10.1016/j.bspc.2016.07.001 . hal-01380875

\section{HAL Id: hal-01380875 \\ https://inria.hal.science/hal-01380875}

Submitted on 13 Oct 2016

HAL is a multi-disciplinary open access archive for the deposit and dissemination of scientific research documents, whether they are published or not. The documents may come from teaching and research institutions in France or abroad, or from public or private research centers.
L'archive ouverte pluridisciplinaire HAL, est destinée au dépôt et à la diffusion de documents scientifiques de niveau recherche, publiés ou non, émanant des établissements d'enseignement et de recherche français ou étrangers, des laboratoires publics ou privés.

$$
\text { Copyright }
$$




\title{
A peak detection method for identifying phase in physiological signals
}

\author{
Nicholas Mitrou*, Alexandre Laurin*, Taylor Dick, and Jessica Inskip \\ Department of Biomedical Physiology \& Kinesiology, Simon Fraser University, Burnaby, \\ BC, Canada
}

Running title: Phase estimation in physiology

Correspondence:

A. Laurin, M3DISIM, Inria, 1 rue Honore d'Estienne d'Orves, Palaiseau, France.

Email: alexandre.laurin@inria.fr

*The first two authors contributed equally to this work. 


\begin{abstract}

\section{Introduction}

To understand the integrated behaviour of biological systems, the interactions between their constituent parts are often studied. For example, the interaction between blood pressure and heart rate reveals information about the cardiac baroreflex. For the purpose of characterising relationships between physiological signals, it is useful to identify phase either as a primary outcome or as an intermediate step to obtain other relevant secondary indices. Existing methods for phase estimation in physiological signals often suffer from a lack of thorough description and standardization which renders reproducibility and interpretation difficult. A relatively simpler peak detection algorithm was compared to the gold-standards of wavelet and Hilbert transforms for its ability to obtain phase.
\end{abstract}

\title{
Methods
}

The accuracy and computation time of the peak detection algorithm was compared to the gold-standard methods in silico by applying all three to data of known phase, and signal-to-noise ratios from -20 to $5 \mathrm{~dB}$. We then compared the performance of the peak detection method to the Hilbert and Wavelet methods by applying each to four different types of in vivo data.

\section{Results}

The peak detection technique is less susceptible to noise and over 10 times faster, computationally, than the wavelet technique. Application to in vivo physiological data shows that equivalent results are obtained from each technique.

\section{Conclusions}

The peak detection method can be used to obtain phase in physiological signals, provide a more clear and direct interpretation, and be more easily reproducible. Because of its design features, peak detection could also be used to identify individual oscillations in relevant signals, as well as to obtain obtain amplitudes and direct time delays. 


\section{Introduction}

Homeostasis is an integral feature of biological systems and is maintained in part by negative feedback loops. The presence of these loops contributes to the creation of oscillations in the corresponding physiological signals. For example, oscillations exist in heart rate (Malik et al., 1996), spontaneous vasomotion of arteries (Mayer, 1876; Fujii et al., 1990), electrical activity of skeletal muscle (Solomonow et al., 1990), and circadian rhythms in hormone levels (Czeisler and Klerman, 1998). The key variables that characterize an oscillation are its amplitude, frequency, and phase. In particular, phase is used to determine the relative progress of one oscillatory cycle at a particular time.

Phase analysis is also applied to the study of the relationship between a pair of associated signals, which can aid understanding of the integrated function of multiple parts of a given system. There are multiple techniques that are routinely employed for estimating the phase of signals, most commonly relying on either a wavelet or Hilbert transform. Both of these techniques are regarded as gold-standards and have been shown to return statistically similar results for signals that have a previously defined narrow frequency band of interest (Le Van Quyen et al., 2001; Quiroga et al., 2002).

The term "phase" is general and can be applied to a wide variety of situations. Since the phase of an in vivo signal can represent the physical state of the system being measured, it is interpreted differently depending on context. For example, in the case of a blood pressure time series, phase can be used as a normalized indicator of arterial contraction and relaxation while in the case of the position of the foot of a pedalling cyclist, phase indicates which part of a particular pedal cycle occurs at any given time. The same type of analysis can be performed on any signal presenting cyclical behaviour, in which each individual cycle (Fig. 1, top) increases phase by $2 \pi$ radians (Fig. 1, middle). Since the circumference of a unit circle is $2 \pi$, phases that differ from one another by a multiple of $2 \pi$ represent the same physiological state. In this study, the convention by which phase is visualised by wrapping it from $-\pi$ to $\pi$ (Fig. 1, bottom) was used.

When two parts of a physiological system interact (as they do, for example in negative feedback loops), oscillations in the signals arising from each system adjust in response to changes in the other 
system. This has been shown in networks in the brain (Doesburg and Ward, 2009), the physiological origin of pathological human tremors (Lauk et al., 1999), the relationship between blood pressure and sway patterns in human standing (Garg et al., 2014), blood flow in adjacent nephrons (Scully et al. 2014), and glycolytic oscillations in cells that receive a periodic nutrient supply (Boiteux et al., 1975). In these cases, phase and phase difference can be used either themselves or as a means to obtain phase lock index, time delay, or other measures of synchrony. Monitoring free-running physiological signals without externally perturbing them, however, cannot distinguish between coincidental synchrony and physical coupling (Pikovsky et al., 2001).

Although the wavelet and Hilbert transforms have been used for decades to study physiological oscillations, methodologies can differ significantly and there remains a lack of clear and detailed guidelines as to their use in various fields. Analyses often rely on 3rd-party software with inconsistent degrees of explicit description. Particularly, imprecise reported methods of filtering and smoothing, either as pre- or post-processing can dramatically alter their output. Because of this, studies are often burdened by lack of reproducibility, and results become difficult to interpret.

The goal of this study was to test the ability of a basic peak detection method to return accurate phase when applied to a variety of in silico and in vivo signals. The aim is that the important simplifications in the proposed methodology would reduce the barriers to transparency and clarity. Because measurement noise and physiological noise are the primary sources of interference with phase estimators, the wavelet, Hilbert, and peak detection techniques were tested for their robustness to noise on simluated signals with known phase. The computing time of these three techniques was also compared. In addition to in silico testing, the phase in signals comprising

four physiological systems were estimated in order to determine phase difference between related pairs of signals. The results obtained with the peak detection method were then compared to those obtained with the wavelet and Hilbert transforms.

\section{Methods}

Each signal, whether in silico or in vivo, was filtered using a $6^{\text {th }}$ order Butterworth bandpass filter prior to analysis. This filter design was chosen for its flat frequency response in the passband, 
a required characteristic when comparing the powers of different frequencies. Filter orders between 6 and 20 had no distinguishable effect on phase relative to each other, and thus the minimum of 6 was chosen to minimize computing time. Signals were first filtered forward then backward to undo any phase alterations that could have been incurred. The passband of the filter was determined case-by-case to isolate relevant signal morphology and is described in detail in the respective sections.

Because phase is defined on a circle, applying arithmetic statistics to it is erroneous. This fact is illustrated using the following example: the mean of the angles $\pi / 4$ and $7 \pi / 4$, calculated arithmetically, is $\pi$. However, since $7 \pi / 4$ is the same as $-\pi / 4$ when plotted on a circle, the mean is 0 . For this reason, circular statistics were used when dealing with phase. A good resource for circular statistics is found in (Batschelet, 1981). When phase difference was shown in figures as a time-series, phase slips of $\pm 2 \pi$ radians, i.e. regions where phase jumped from $\pi$ to $-\pi$ only because of wrapping, were removed for clarity of presentation.

All data synthesis and analysis was performed using Matlab (r2014b, The Mathworks, Natick, MA, USA) and statistical comparisons were performed using SPSS (v22, IBM, Armonk, NY, USA). Results are expressed as mean \pm standard deviation unless otherwise indicated, and differences were considered significant if $p<0.05$.

\section{Estimating phase}

This section describes the wavelet transform, Hilbert transform, and peak detection techniques. To allow comparisons between the techniques on an even playing field, their pre- and post-processing methods were unified as much as possible. To eliminate edge effects, $3 / f_{L}$ s were removed from both ends of all phase time-series, where $f_{L}$ was the low-frequency bound of the filter passband.

i The Morlet wavelet transform is a Gaussian-windowed Fourier transform. The Morlet wavelet of order six, used in this study, is a cosine wave modulated by a Gaussian of such width that six periods fit in $95 \%$ of its area. The wavelet transform returns power, a measurement of how well a wavelet represents the given signal on the given time interval. It then shifts that wavelet in time and frequency, to generate the power spectrum of the signal over time. For a great 
introduction on the subject see (Farge, 1992), and (Torrence and Compo, 1998). The software used for these analyses is freely available (http://paos.colorado.edu/research/wavelets/ software.html).

To illustrate, a cosine wave of frequency $\alpha$ during a certain time is expected to have a high wavelet transform magnitude at that time and frequency. Conversely, the wavelet transform of a random noisy signal would have a relatively low magnitude for all times and frequencies. In this study, phase was determined for the the frequency $\alpha_{\max }$ with the highest power for every time point $t_{i}$. Phase was computed as

$$
\phi\left(t_{i}\right)=\tan ^{-1} \frac{\operatorname{im}\left(s_{w}\left(\alpha_{\max }, t_{i}\right)\right)}{\operatorname{re}\left(s_{w}\left(\alpha_{\max }, t_{i}\right)\right)}
$$

where $s_{w}$ is the wavelet transform of the signal $s$.

ii The Hilbert transform uses the Fourier transform to create a $90^{\circ}$ phase shift filter. As it pertains to this study, it obtains phase information by using the Fourier power to compute a weighted average of the phases in the frequency domain

$$
\phi=\frac{1}{\sum_{\alpha}\left|s_{F}(\alpha)\right|} \sum_{\alpha}\left|s_{F}(\alpha)\right| \cdot \tan ^{-1} \frac{\operatorname{im}\left(s_{F}(\alpha)\right)}{\operatorname{re}\left(s_{F}(\alpha)\right)},
$$

where $s_{F}$ is the Fourier transform of the signal $s$.

iii The peak detection algorithm identifies all local maxima higher than 0 on the filtered signal. Because of the nature of the filter, no peaks can occur closer than $1 / f_{H}$ s from each other, where $f / H$ is the high-frequency bound of the filter passband. Physiologically, this minimum gap represents the smallest possible distance between two successive peak events. A point is identified as a local maxima in the simplest possible sense, i.e. if it is higher than both the directly previous and directly successive points.

If $t_{a}$ and $t_{b}$ were the timings of two successive identified peaks, then the phase $\phi$ between these 
peaks is defined as

$$
\phi(t)=2 \pi \frac{t-t_{a}}{t_{b}-t_{a}}, \quad t_{a}<t \leq t_{b}
$$

\section{In silico analyses}

The peak detection phase-estimation technique was compared to the two other gold-standard techniques in silico. The main goal was to compare their accuracy with respect to signal-to-noise (SNR) ratio, but also of interest was their computing time.

The in silico data were designed to resemble the physiological data considered later in this manuscript. To this effect, individual sinusoidal cycles with different frequencies $\left\{f_{i}\right\}$ were joined. To mimic the tendency of physiological oscillations to occur around a central frequency, this set of frequencies $\left\{f_{i}\right\}$ was created as a normal distribution $f_{i}(\mu, \sigma)$ around a central frequency $\mu=0.1$ $\mathrm{Hz}$, and standard deviation $\sigma=0.01$. Additionally, the signal was designed to change frequency every cycle, have a sample frequency of $100 \mathrm{~Hz}$, and create 7 minutes of signal (Figure 2A). The $100 \mathrm{~Hz}$ sample frequency was chosen to be well above the Nyquist frequency.

Noise was added to each simulation time series for signal-to-noise ratios SNR $\in[-20,5] \mathrm{dB}$, in $1 \mathrm{~dB}$ increments (Figure 2B). At each SNR, the phase was estimated using the three techniques and the difference between the true phase and estimated phase was computed. This process was repeated 100 times (Figure 3. Table1). The computation time was measured for each trial (Table 2).

To determine differences in the precision of each technique, the difference between mean and squared residuals of phase at each SNR, compared to true phase, was tested using a parametric Watson-Williams multi-sample test for equal means, which can be used as a one-way ANOVA test for circular data (Zar et al. 1999$)$. For any particular SNR, if the maximum absolute residual was less than 0.1 radians, the differences therein were not considered to be of potential physiological relevance and were therefore not included in our statistical comparison. Squared residuals were not different between Hilbert and peak detection at any SNR, while the wavelet values were different from the other two techniques when SNR was less than $-9 \mathrm{~dB}$.

Noise in physiological signals can either come from data acquisition or be intrinsic to the system itself. While the former can be estimated using standard techniques, estimates for the latter are 
usually much harder to obtain. In a blood pressure measurement, for example, an unreported involuntary muscle contraction in a subject could create noise as a temporary oscillation in a relevant frequency band, which would be difficult to quantify. While an SNR of $-9 \mathrm{~dB}$ is very low, some types of physiological can be that noisy and still contain relevant information (Scully et al. 2013).

\section{In vivo analyses}

The three phase estimation techniques were applied to four sets of physiological data. For each set, the acquisition and formatting techniques are described. The animal experiments were conducted according to the guidelines set forth by the Canadian Council on Animal Care and approved by the Animal Care Committee of Simon Fraser University. Human experiments were conducted in accordance with the Declaration of Helsinki and with approval from the Simon Fraser University Office of Research Ethics.

To determine the validity of phase difference obtained by the peak detection technique, a parametric Watson-Williams multi-sample test for equal means was performed on each pair of result sets.

\section{Human systolic blood pressure and RR intervals}

The phase difference between systolic blood pressure (SBP) and RR intervals is used to estimate the time delay of efferent cardiac baroreflex control (Halámek et al., 2003). Eight healthy subjects (5 males) ages 20-57, were tested in the supine position. Blood pressure and electrocardiogram (ECG, lead II configuration) were recorded using the Finometer (Finapres Medical Systems, Amsterdam, Netherlands) for five minutes at a sample frequency of $1 \mathrm{kHz} . \mathrm{R}$ waves and SBP peaks were identified, RR intervals were computed, and the resulting time series were resampled at $5 \mathrm{~Hz}$. SBP and RR interval time series were filtered with a passband of $[0.04,0.15] \mathrm{Hz}$ to isolate low-frequency variability (Malik et al. 1996). Oscillations in this band are present in both RR intervals and SBP, correspond to each other, and are called Mayer waves in SBP (Mayer, 1876). The phase of both signals was computed using the three techniques, showing the expected phase lag between SBP and 
RR intervals (Figure 4).

For each subject, the circular mean and standard deviation of phase difference between SBP and RR intervals was computed. The mean phase difference over all participants was $-1.91 \pm 0.74$ $\mathrm{rad}$ for the wavelet technique, $-1.70 \pm 0.34 \mathrm{rad}$ for the Hilbert technique, and $-1.53 \pm 0.31 \mathrm{rad}$ for the peak detection technique (Figure 5). For estimation of cardiac baroreflex phase difference, values form all three approaches were not statistically different $(p>0.64)$.

\section{Human nasal temperature and RR intervals}

Changes in thoracic pressure caused by respiration affect $R R$ intervals eliciting respiratory sinus arrhythmia. This phenomenon can be investigated by measuring the phase difference between nasal temperature (NT) and RR intervals. Seventeen healthy participants (7 males) ages 18-51, with no experience in meditation were tested. Participants were seated, instructed to close their eyes, and to remain motionless. For 7 minutes, they were instructed to remain silent and listen to recorded talk radio.

Nasal temperature and ECG (lead II configuration) were recorded using the Pneumocard (Baevsky et al., 2009). RR interval time-series were obtained from the ECG in the same manner as described above. NT was resampled to $5 \mathrm{~Hz}$ and used to determine breathing rate. NT and $\mathrm{RR}$ interval time series were filtered to a passband of $[0.15,0.4] \mathrm{Hz}$ to isolate high-frequency variability (Malik et al. 1996). Heart rate variability in this high-frequency range is caused by respiratory sinus arrhythmia and the central frequency represents the respiratory frequency (Gerhardt, 1877). The phase of both signals was computed using the three techniques, showing the expected phase lag between NT and RR intervals (Figure 6).

For each subject, the circular mean and standard deviation of phase difference between NT and RR intervals was computed. The mean phase difference for all participants was $-0.7 \pm 0.37 \mathrm{rad}$ for the wavelet technique, $-0.73 \pm 0.35 \mathrm{rad}$ for the Hilbert technique, and $-0.81 \pm 0.48$ rad for the peak detection technique (Figure 7). For estimation of phase differences associated with respiratory sinus arrhythmia, values form all three approaches were not statistically different $(p>0.54)$. 
Human electromyograms and muscle-tendon length

Mechanical function largely depends on the timing of muscle activation relative to muscle lengthening or shortening (Dickinson et al. 2000). The phase of muscle activation relative to muscle length change has emerged as an important variable by which the nervous system can regulate mechanical performance of muscles during locomotion. Eight cyclists were tested on an indoor cycle ergometer (Indoor Trainer, SRM, Julich, Germany). Limb kinematics were collected using an optical motion capture system (Certus Optotrak, NDI, Waterloo, Canada). LED markers were placed on the pelvis, greater trochanter, thigh segment (triad), femoral lateral epicondyle, shank (triad) lateral malleolus, calcaneus, metatarsophalangeal joint, and the bike pedal of the left and right limbs. Participants pedalled at a cadence of 80 revolutions per minute. Time varying medial gastrocnemius (MG) muscle-tendon unit lengths were calculated using a subject specific scaled musculoskeletal model (pre-defined muscle paths from cadaveric studies) and experimentally collected joint kinematics in the biomechanics simulation software OpenSim (Delp et al., 2007).

Surface EMG was recorded during the cycling conditions from the $\mathrm{MG}$ using bipolar $\mathrm{Ag} / \mathrm{AgCl}$ electrodes (10 mm diameter, $21 \mathrm{~mm}$ spacing) and Biovision amplifiers (Biovision, Wehrheim, Germany). Electrodes were placed in the mid-region of the muscle bellies after the hair had been removed and the skin cleaned with isopropyl alcohol solution. EMG was sampled at $2 \mathrm{kHz}$ and recorded using a 16-bit analogue-to-digital convertor (USB-6210, National Instruments, Austin, TX, USA). EMG signals were quantified by their intensities during each pedal revolution. The intensity is a close approximation to the power of the signal and was calculated across the frequency band $[10,450] \mathrm{Hz}$ using an EMG-specific wavelet analysis (von Tscharner, 2000). All 14-second time series were downsampled to $100 \mathrm{~Hz}$ and filtered to isolate the frequency of pedalling using a passband of $[1.0,1.5] \mathrm{Hz}$. The phase of both signals was computed using the three techniques, displaying a phase lag between muscle activity (EMG intensity) and the onset of muscle-tendon unit shortening (Fig. 8) consistent with previous findings (Dickinson et al., 2000).

For each subject, the circular mean and standard deviation of phase difference between muscle activity (EMG intensity) and the onset of muscle-tendon unit shortening was computed. The mean phase difference for all participants was $-0.50 \pm 0.96 \mathrm{rad}$ for the wavelet technique, $-0.50 \pm 0.96$ 
rad for the Hilbert technique, and $-0.50 \pm 0.96 \mathrm{rad}$ for the peak detection technique (Fig. 9). For estimation of phase difference between muscle activity and onset of muscle shortening, values form all three approaches were not statistically different $(p>0.99)$.

\section{Synchrony of renal autoregulation}

It has been shown previously that laser speckle perfusion imaging (LSCI) can detect physiological signals including those associated with renal autoregulation (Scully et al., 2014), and that these dynamics can be segmented into regions that are phase-synchronised (Brazhe et al., 2014, Scully et al., 2014). Autoregulation is mediated in part by the classic myogenic response (MR) of smooth muscle, where increased intraluminal pressure causes vasoconstriction, and decreased pressure causes vasodilation. Male Long-Evans rats $(n=8)$ were anesthetised with isoflurane and their left kidney was exposed via a subcostal flank incision. Cortical perfusion was monitored for 5 minutes on approximately $1 / 3$ of the visible surface of the kidney with LSCI using a Moor full-field laser speckle perfusion imager (Moor Instruments, Axminster, UK).

Three regions of interest (ROI) were selected on the surface. ROI1 and ROI2 were the regions with the highest total flux in the bottom-left tenth of the total area, while ROI3 was the region with the highest total flux in the upper-right tenth of the total area. ROI1 and ROI2 were between 361 and $424 \mu \mathrm{m}$ apart while ROI3 was located $2.36 \mathrm{~mm}$ away from ROI1. Perfusion time series were extracted from each ROI. Each $25 \mathrm{~Hz}$ time series was downsampled to $1 \mathrm{~Hz}$ filtered to isolate the frequency range associated with $\mathrm{MR}$ in rats using a passband of [0.09, 0.32] Hz. The phase of each ROI was computed using the three techniques (Fig. 10).

For each rat, the circular mean and standard deviation of phase difference between the two nearby and the two far ROI were computed. In nearby ROI the mean phase difference for all rats was $-0.03 \pm 0.13 \mathrm{rad}$ for the wavelet technique, $-0.04 \pm 0.10 \mathrm{rad}$ for the Hilbert technique, and $0.03 \pm 0.12 \mathrm{rad}$ for the peak detection technique (Fig. 11). In far ROI the mean phase difference for all rats was $-0.30 \pm 0.55 \mathrm{rad}$ for the wavelet technique, $-0.26 \pm 0.40 \mathrm{rad}$ for the Hilbert technique, and $-0.19 \pm 0.49 \mathrm{rad}$ for the peak detection technique (Fig. 12). For estimation of phase difference between blood perfusion in different regions of a rat kidney, all three approaches were 
not statistically different $(p>0.72)$.

\section{Discussion}

A basic peak detection technique was compared to gold-standard wavelet and Hilbert transform techniques for estimating phase in terms of accuracy and computing time. The Morlet wavelet transform technique obtained phase at each time point using the frequency of highest power. The Hilbert transform technique obtained phase by performing a weighted average of the phase of each frequency using the Fourier transform. The peak detection technique identified local maxima and interpolated phase from them.

Upon testing these techniques in silico, their behaviours under signal-to-noise ratios from $[-20,5]$ dB were distinguished. The wavelet technique was the most susceptible to increasing levels of noise, resulting in different phase estimates for SNR lower than $-9 \mathrm{~dB}$. The Hilbert transform and peak detection techniques continued to perform well, even with SNR below $-9 \mathrm{~dB}$. Under conditions of very high noise levels, therefore, the peak detection technique performed as well or better than the gold-standard techniques. It is likely that the wavelet technique, as designed here, was more susceptible to noise because it identified a single frequency as the carrier of information, rather than use a weighted average, as in the Hilbert transform technique.

The wavelet technique took the most computing time - greater than 10 times the duration of the other two. The only difference in the implementation of each phase estimation algorithm was the phase estimator itself, all pre- and post-processing being identical. This shows that the peak detection technique is more accurate and faster than the wavelet techniques under these conditions. Greater computing speed could be of benefit in the case of especially large data sets.

It should be acknowledged that the wavelet transform comprises an expansive tool that was used here to obtain a single specific quantity. In effect, the algorithm computed vastly more information than was actually needed, and then discarded it. Whereas the Hilbert transform and peak detection techniques depend critically on a priori knowledge of the relevant frequency domain, the wavelet technique implicitly computes a time-frequency representation of the signal, and therefore could easily be adapted to work without any pre-filtering. Furthermore, only the Morlet wavelet of 
order 6 was tested. In certain cases, it is known that specially designed wavelet banks are able to return more accurate results than generic ones (von Tscharner, 2000). The wavelet technique, then, presents many advantages that were not employed or tested in this study.

Although the simulated data was designed to resemble physiological signals, it presents clear limitations. Most notably, physiological data is almost always comprised of stochastic components. The comparisons as they were made in this study are therefore unable to to distinguish the robustness of the three techniques under varying conditions of stochasticity.

When applied to pairs of physiological signals, all three techniques returned statistically equivalent phase difference values. This is consistent with the claim that the peak detection technique could be used to obtain valid phase in various physiological contexts.

The main distinction of the peak detection technique is that it is not limited by the timefrequency uncertainty principle. When using frequency analyses such as the Hilbert or wavelet transforms, it is impossible to reduce the time spread and frequency spread simultaneously Hardy (1933). The peak detection technique extrapolates phase information from time information under the assumption that measurable rises and drops occur in a strictly known frequency band.

The success of the peak detection method has a number of useful implications in physiological signal analysis. Firstly, accurate estimation of phase implies an accurate estimation of other relevant frequency domain information. For example, 'instantaneous' angular frequency could be obtained from the derivative of phase. It would also be possible to measure the amplitude of the peaks to obtain a analog for power or energy in the relevant passband. An accurate estimation of phase from peak detection is also consistent with the hypothesis that the implicit division of the signal into individual oscillation cycles is meaningful. In other words, if cutting up a signal in peak-topeak segments returns valid phase, then the segments themselves might be a valid representation of single oscillations. The accurate identification of single oscillations could prove quite useful in contexts where variables therein could be measured and interpreted. For example, in the case of low-frequency oscillations in blood pressure and RR intervals, the amplitudes, time between peaks, and slopes of single cycles could provide useful information about baroreflex sensitivity.

It could be possible to further improve the accuracy of the peak detection technique by iden- 
tifying additional features in the analysed signal. In this study, only peaks were identified, but it would be straightforward to also identify nadirs in the signal itself as well as in its derivative.

The peak detection technique is computationally simpler than the wavelet and Hilbert transform techniques while remaining as accurate. Its relative simplicity allows for better reproducibility and comparison of results, as well as simpler physiological interpretation. There are, however, a number of hypothetical cases where the morphology of a signal would prevent its application. One can imagine a signal constituting plateaux (flat maximal regions), which would prevent clear identification of a single peak per cycle. To a lesser degree, oscillations that are skewed, e.g. a signal that drops quickly and rises slowly, would also create errors in phase estimation from peak detection.

\section{Conclusion}

Existing methods for phase estimation in physiological signals often suffer from a lack of thorough description and standardization which renders reproducibility and interpretation difficult. This study's results are consistent with the claim that the described peak detection technique can be used to obtain phase in a variety of physiological signals Because of its design features, results obtained from the peak detection technoque provide a more clear and direct interpretation, and are more easily reproducible. There remains work to be done to design peak detection analogs to coherence and gain, and study the potential of identifying individual oscillations in relevant data.

\section{Acknowledgments}

The authors would like to thank Dr. Will Cupples, Dr. Andrew Blaber, Dr. James Wakeling, and Dr. Victoria Claydon, our respective PhD supervisors, for their support and guidance. Data were collected under the auspices of Canadian Institue of Health Research grant MOP-102694 to W. Cupples, the Aerospace Physiology projects account, a National Sciences and Engineering Research Council [261262-2008] to J. Wakeling, a Heart and Stroke Foundation of British Columbia and the

Yukon grant awarded to V. Claydon. J. Inskip was supported by a CIHR doctoral award. T. Dick 
was supported by a NSERC post-graduate scholarship.

\title{
Conflict of interest
}

\author{
None.
}

\section{References}

R. Baevsky, I. Funtova, A. Diedrich, A. Chernikova, J. Drescher, V. Baranov, and J. Tank. Autonomic function testing aboard the ISS using "PNEUMOCARD". Acta Astronautica, 65(7): 930-932, 2009.

E. Batschelet. Circular statistics in biology, volume 371. Academic Press London, 1981.

A. Boiteux, A. Goldbeter, and B. Hess. Control of oscillating glycolysis of yeast by stochastic, periodic, and steady source of substrate: a model and experimental study. Proceedings of the National Academy of Sciences, 72(10):3829-3833, 1975.

A. R. Brazhe, D. J. Marsh, N.-H. Holstein-Rathlou, and O. Sosnovtseva. Synchronized renal blood flow dynamics mapped with wavelet analysis of laser speckle flowmetry data. PloS one, 9(9): e105879, 2014.

C. A. Czeisler and E. B. Klerman. Circadian and sleep-dependent regulation of hormone release in humans. Recent Progress in Hormone Research, 54:97-130, 1998.

S. L. Delp, F. C. Anderson, A. S. Arnold, P. Loan, A. Habib, C. T. John, E. Guendelman, and D. G. Thelen. Opensim: open-source software to create and analyze dynamic simulations of movement. Biomedical Engineering, IEEE Transactions on, 54(11):1940-1950, 2007.

M. H. Dickinson, C. T. Farley, R. J. Full, M. Koehl, R. Kram, and S. Lehman. How animals move: an integrative view. Science, 288(5463):100-106, 2000. 
S. M. Doesburg and L. M. Ward. Synchronization between sources: emerging methods for understanding large-scale functional networks in the human brain. In Coordinated Activity in the Brain, pages 25-42. Springer, 2009.

M. Farge. Wavelet transforms and their applications to turbulence. Annual Review of Fluid Mechanics, 24(1):395-458, 1992.

K. Fujii, D. D. Heistad, and F. M. Faraci. Vasomotion of basilar arteries in vivo. American Journal of Physiology: Heart $\& 3$ Circulatory Physiology, 258(6 Pt 2):H1829-34, 1990.

A. Garg, D. Xu, A. Laurin, and A. P. Blaber. Physiological interdependence of the cardiovascular and postural control systems under orthostatic stress. American Journal of Physiology-Heart and Circulatory Physiology, 307(2):H259-H264, 2014.

C. A. C. J. Gerhardt. Handbuch der Kinderkrankheiten: Krankheiten der Neugeborenen, Allgemeinerkrankungen erster Theil (Acute Infectionskrankheiten), volume 2. H. Laupp'schen Buchhandlung, 1877.

J. Halámek, T. Kára, P. Jurák, M. Souček, D. P. Francis, L. C. Davies, W. K. Shen, A. J. S. Coats, M. Novák, Z. Nováková, R. Panovský, J. Toman, J. Šumbera, and V. K. Somers. Variability of phase shift between blood pressure and heart rate fluctuations a marker of short-term circulation control. Circulation, 108(3):292-297, 2003.

G. H. Hardy. A theorem concerning Fourier transforms. Journal of the London Mathematical Society, s1-8(3):227-231, 1933.

M. Lauk, B. Köster, J. Timmer, B. Guschlbauer, G. Deuschl, and C. Lücking. Side-to-side correlation of muscle activity in physiological and pathological human tremors. Clinical Neurophysiology, 110(10):1774-1783, 1999.

M. Le Van Quyen, J. Foucher, J.-P. Lachaux, E. Rodriguez, A. Lutz, J. Martinerie, and F. J. Varela. Comparison of hilbert transform and wavelet methods for the analysis of neuronal synchrony. Journal of Neuroscience Methods, 111(2):83-98, 2001. 
M. Malik, J. T. Bigger, A. J. Camm, R. E. Kleiger, A. Malliani, A. J. Moss, and P. J. Schwartz. Heart rate variability standards of measurement, physiological interpretation, and clinical use. European Heart Journal, 17(3):354-381, 1996.

S. Mayer. Studien zur physiologie des herzens und der blutgefässe 6. abhandlung: Über spontane blutdruckschwankungen. Sitzungsberichte Akademie der Wissenschaften in Wien. Mathematischnaturwissenschaftliche Classe, Anatomie, 74:281-307, 1876.

A. Pikovsky, M. Rosenblum, and J. Kurths. Synchronization: A universal concept in nonlinear sciences. Cambridge University Press, 2001.

R. Q. Quiroga, A. Kraskov, T. Kreuz, and P. Grassberger. Performance of different synchronization measures in real data: a case study on electroencephalographic signals. Physical Review E, 65 (4):041903, 2002.

C. Scully, N. Mitrou, B. Braam, W. Cupples, and K. Chon. Segmentation of renal perfusion signals from laser speckle imaging into clusters with phase synchronized dynamics. IEEE Transactions on Biomedical Engineering, 61(7), 2014.

C. G. Scully, N. Mitrou, B. Braam, W. A. Cupples, and K. H. Chon. Detecting physiological systems with laser speckle perfusion imaging of the renal cortex. American Journal of PhysiologyRegulatory, Integrative and Comparative Physiology, 304(11):R929-R939, 2013.

M. Solomonow, C. Baten, J. Smit, R. Baratta, H. Hermens, R. D’Ambrosia, and H. Shoji. Electromyogram power spectra frequencies associated with motor unit recruitment strategies. Journal of Applied Physiology, 68(3):1177-85, 1990.

C. Torrence and G. P. Compo. A practical guide to wavelet analysis. Bulletin of the American Meteorological Society, 79(1):61-78, 1998.

V. von Tscharner. Intensity analysis in time-frequency space of surface myoelectric signals by wavelets of specified resolution. Journal of Electromyography and Kinesiology, 10(6):433-445, 2000. 
J. H. Zar et al. Biostatistical analysis. Pearson Education India, 1999.

Table 1: Difference between mean estimated phase and true phase of a simulated oscillating signal. The difference was computed at each level of SNR from -20 to $5 \mathrm{~dB}$, and this was repeated 100 times.

\begin{tabular}{ccccc}
\hline SNR $(\mathrm{dB})$ & Wavelet & \multicolumn{1}{c}{ Hilbert } & Peak Det. \\
\hline-20 & $-0.3 \pm 0.1 *$ & $0.01 \quad \pm 0.01$ & $0.004 \pm 0.002$ \\
-15 & $0.06 \pm 0.05 *$ & $0.03 \pm 0.01$ & $0.002 \pm 0.001$ \\
-10 & $-0.011 \pm 0.010^{*}$ & $-0.002 \pm 0.004$ & $0.003 \pm 0.001$ \\
-5 & $0.003 \pm 0.001$ & $0.006 \pm 0.002$ & $0.002 \pm 0.001$ \\
0 & $-0.002 \pm 0.001$ & $-0.005 \pm 0.063$ & $0.0030 \pm 0.0003$ \\
5 & $-0.002 \pm 0.001$ & $0.007 \pm 0.056$ & $0.0020 \pm 0.0002$ \\
$\infty$ & $0.002 \pm 0.001$ & $0.0060 \pm 0.0002$ & $0.0010 \pm 0.0001$ \\
\hline \multicolumn{5}{c}{ Mean \pm standard deviation } \\
& $* 0.05$ compared to Hilbert and peak detection
\end{tabular}

Table 2: Mean computation time for each technique of phase estimation for each trial with SNR ranging from -20 to $5 \mathrm{~dB}$, with 100 simulations.

\begin{tabular}{cc}
\hline Method & Time $(\mathrm{s})$ \\
\hline Wavelet & $1.7288 \pm 0.0180$ \\
Hilbert & $0.0877 \pm 0.0314^{*}$ \\
Peak Det. & $0.1364 \pm 0.0671^{*}$ \\
\hline Mean \pm standard deviation. \\
${ }^{*} p<0.05$ compared to wavelet
\end{tabular}



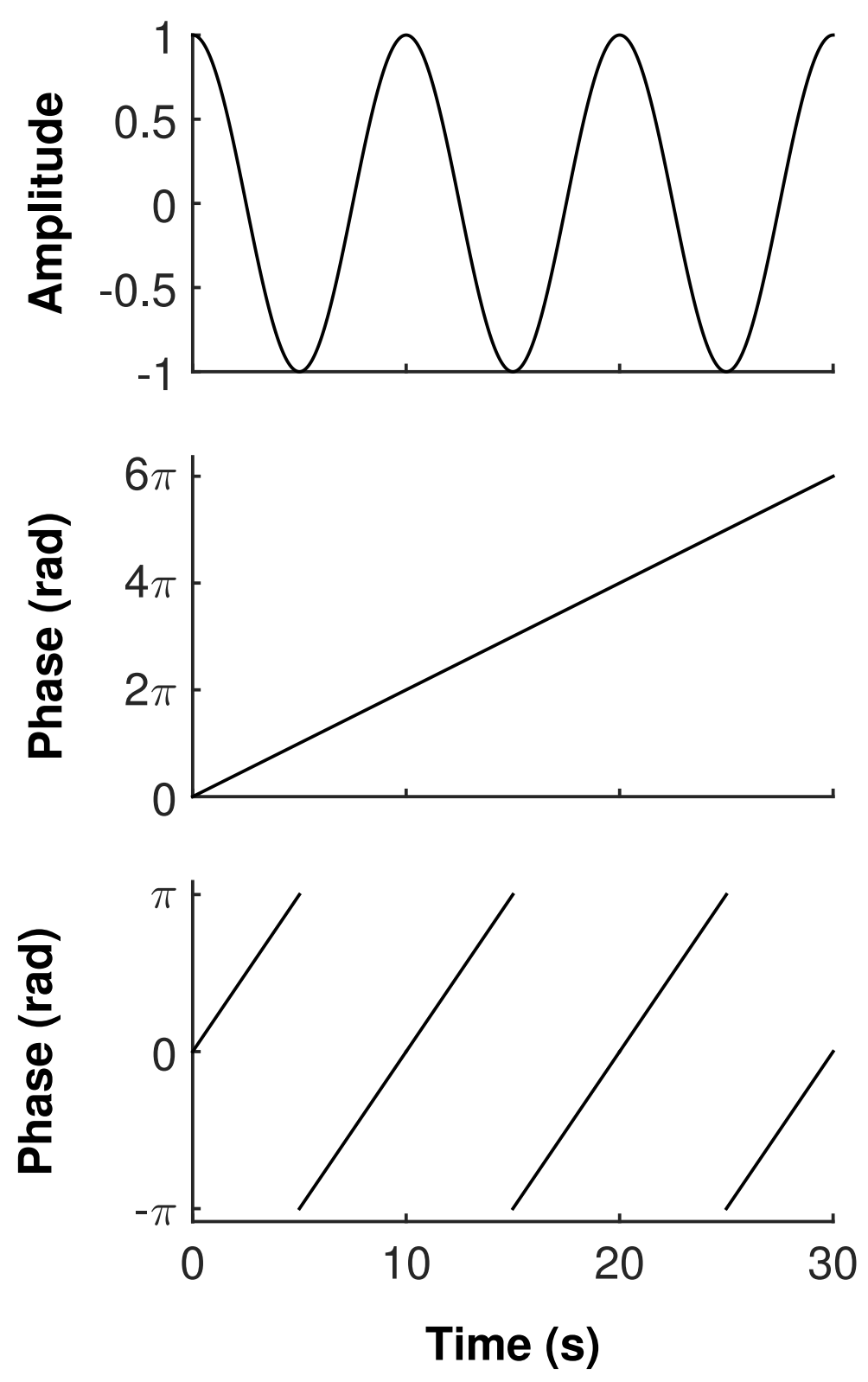

Figure 1: Example cosine function and its corresponding phase. There are three cycles of the cosine wave (top), resulting in a total increase of $6 \pi$ radians of phase (middle). The bottom panel shows the same phase wrapped from $-\pi$ to $\pi$. 

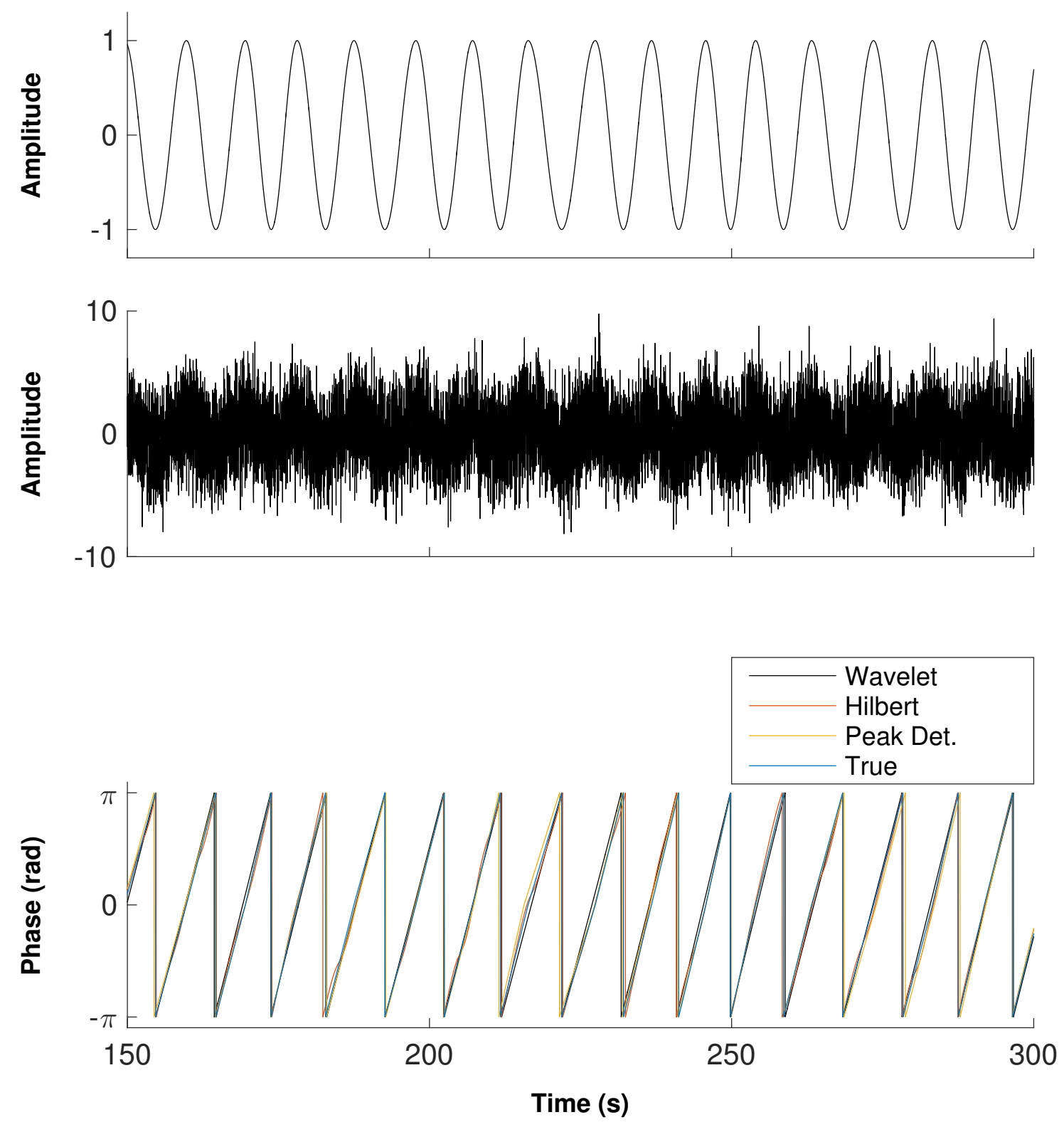

Figure 2: Example signals and phase estimates, in silico. Top: Clean signal composed of joined sinusoids of varying frequencies with central frequency $\mu=0.1 \mathrm{~Hz}$, and frequency standard deviation $\sigma=0.01 \mathrm{~Hz}$. Signal-to-noise ratio $(\mathrm{SNR})=\infty$. Middle: A noisy signal. SNR $=-10 \mathrm{~dB}$. Bottom: Wrapped phase estimates for wavelet, Hilbert, and peak detection techniques at $\mathrm{SNR}=-10 \mathrm{~dB}$. 


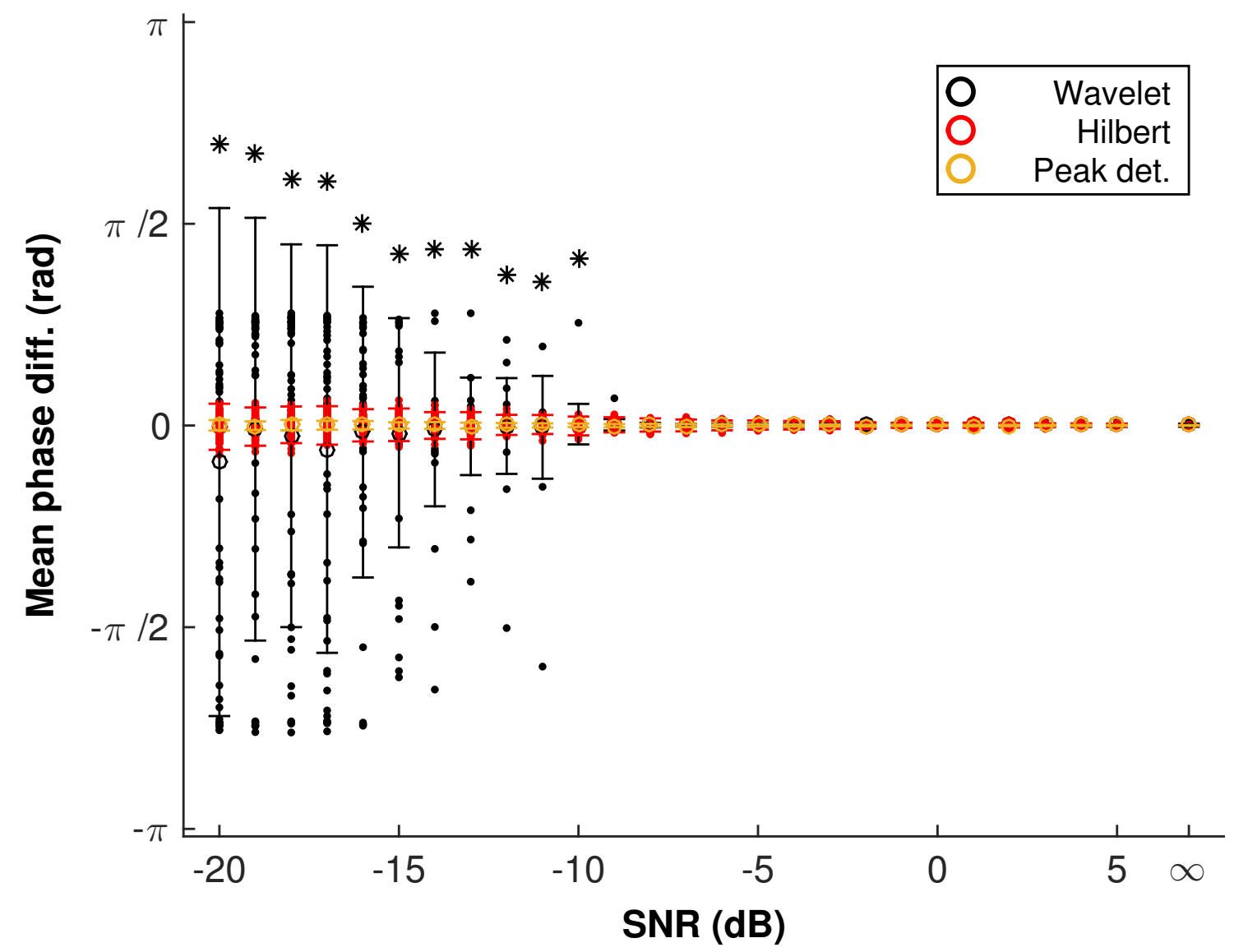

Figure 3: The effect of added Gaussian white noise on the accuracy of each phase estimator. At each signal-to-noise ratio (SNR), the difference between true phase and estimated phase is shown as a solid point for each of the 100 trials. The mean difference is shown as an open circle and error bars are $1.96 \times$ standard deviation. ${ }^{*}$ Mean wavelet phase difference is significantly different from both other techniques' mean $(\mathrm{p}<0.05)$. 

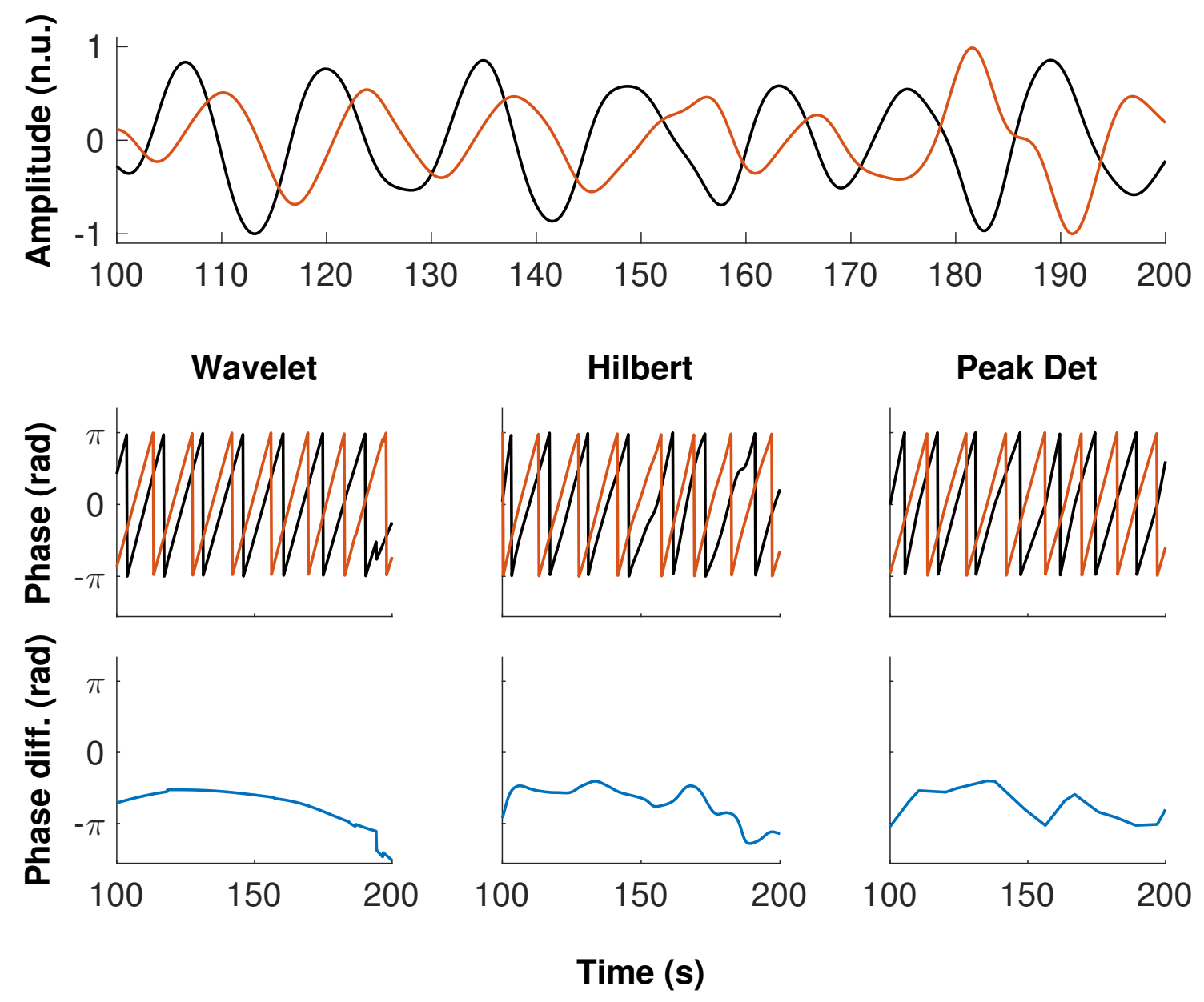

Figure 4: Top: Example filtered RR interval (red) and systolic blood pressure (SBP, black) time series. Static oscillations in SBP around a frequency of $0.1 \mathrm{~Hz}$ create analogous oscillations in RR intervals. Normalised units were obtained by subtracting the mean and dividing by the resulting maximum amplitude. Middle:The phase of SBP and RR intervals are shown as calculated by each technique. Bottom: Phase difference between the two time series. 
Wavelet
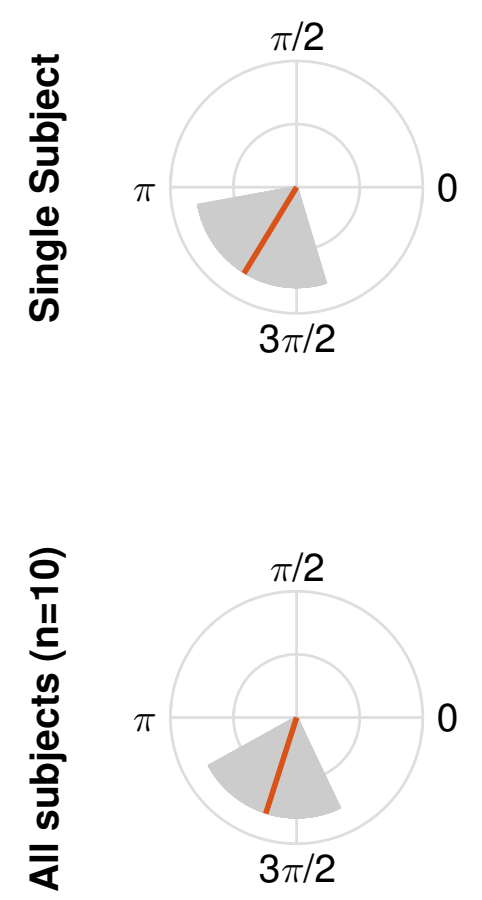

Hilbert
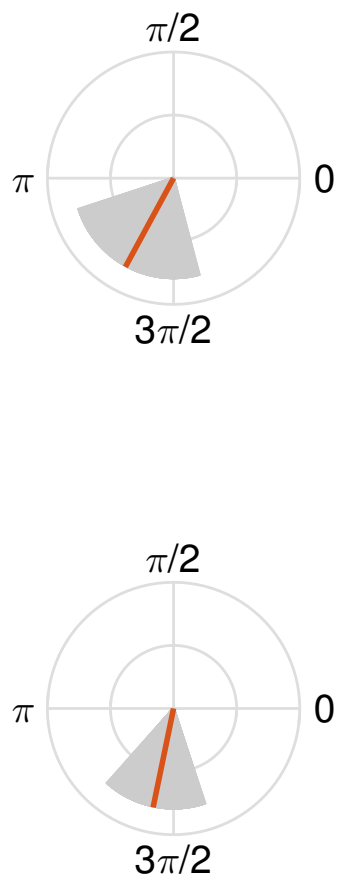

Peak Det
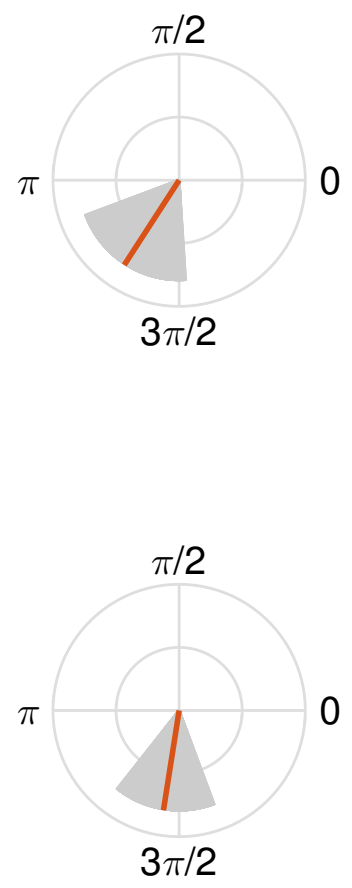

Figure 5: Top: Example circular mean (red line) and circular standard deviation (grey) of phase difference between RR intervals and systolic blood pressure (SBP) for the three techniques in one individual. Bottom: Group circular mean (red) and standard deviation (grey) of circular means $(n=10)$ of phase difference between RR intervals and SBP. All pairs of distributions were not statistically different $(p>0.64)$. 

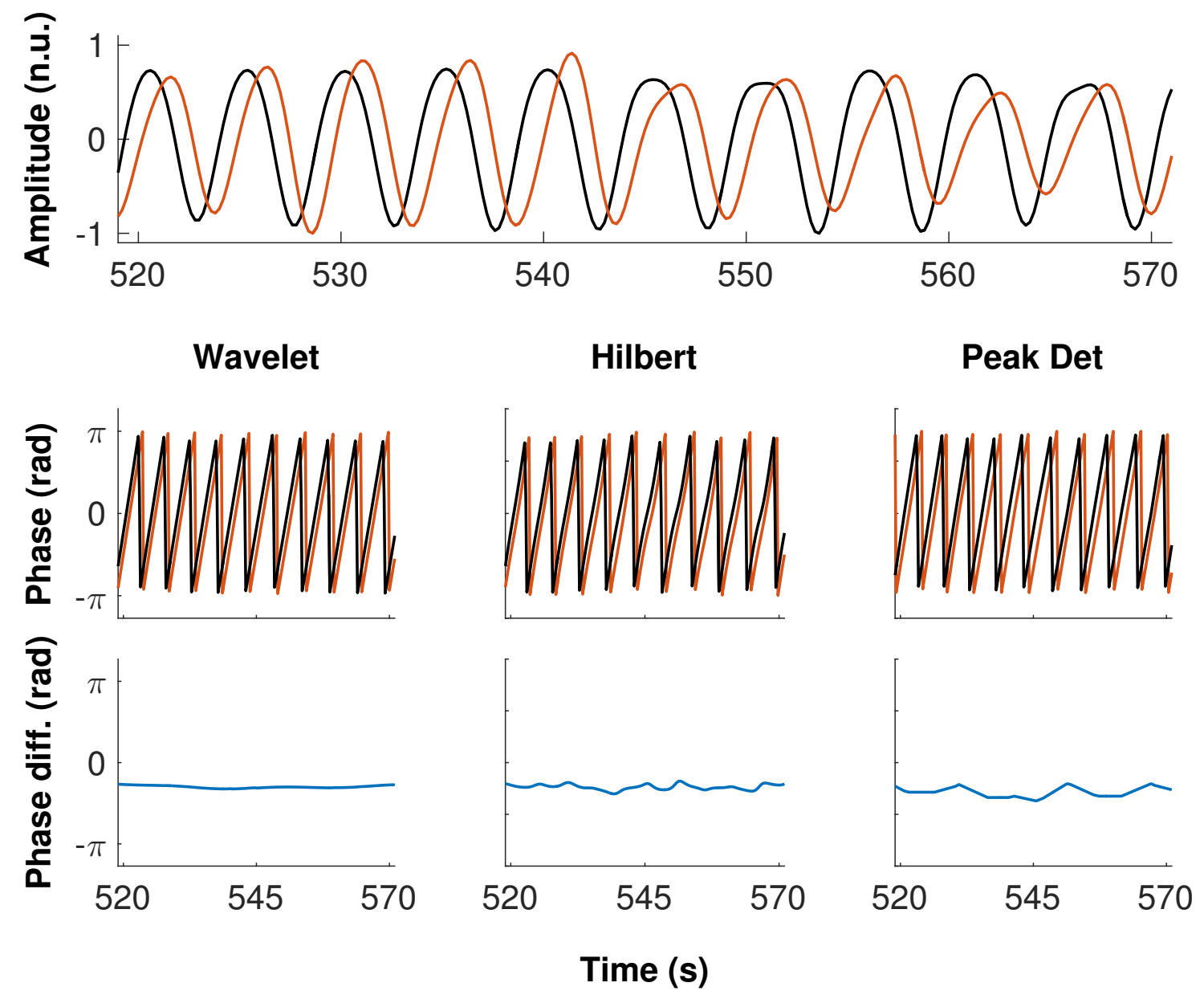

Figure 6: Top: Example filtered nasal temperature (NT, red) and RR intervals (black) time series. Changes in thoracic pressure caused by respiration $(\approx 0.25 \mathrm{~Hz})$ affect $\mathrm{RR}$ intervals, eliciting respiratory sinus arrhythmia. Normalised units were obtained by subtracting the mean and dividing by the resulting maximum amplitude. Middle: The phase of NT and RR intervals are shown as calculated by each technique. Bottom: Phase difference between the two time series. 

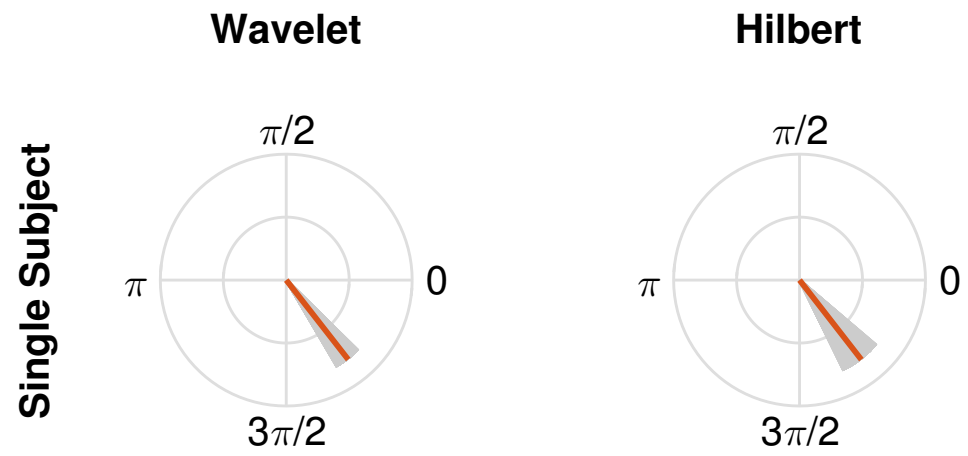

\section{Peak Det}
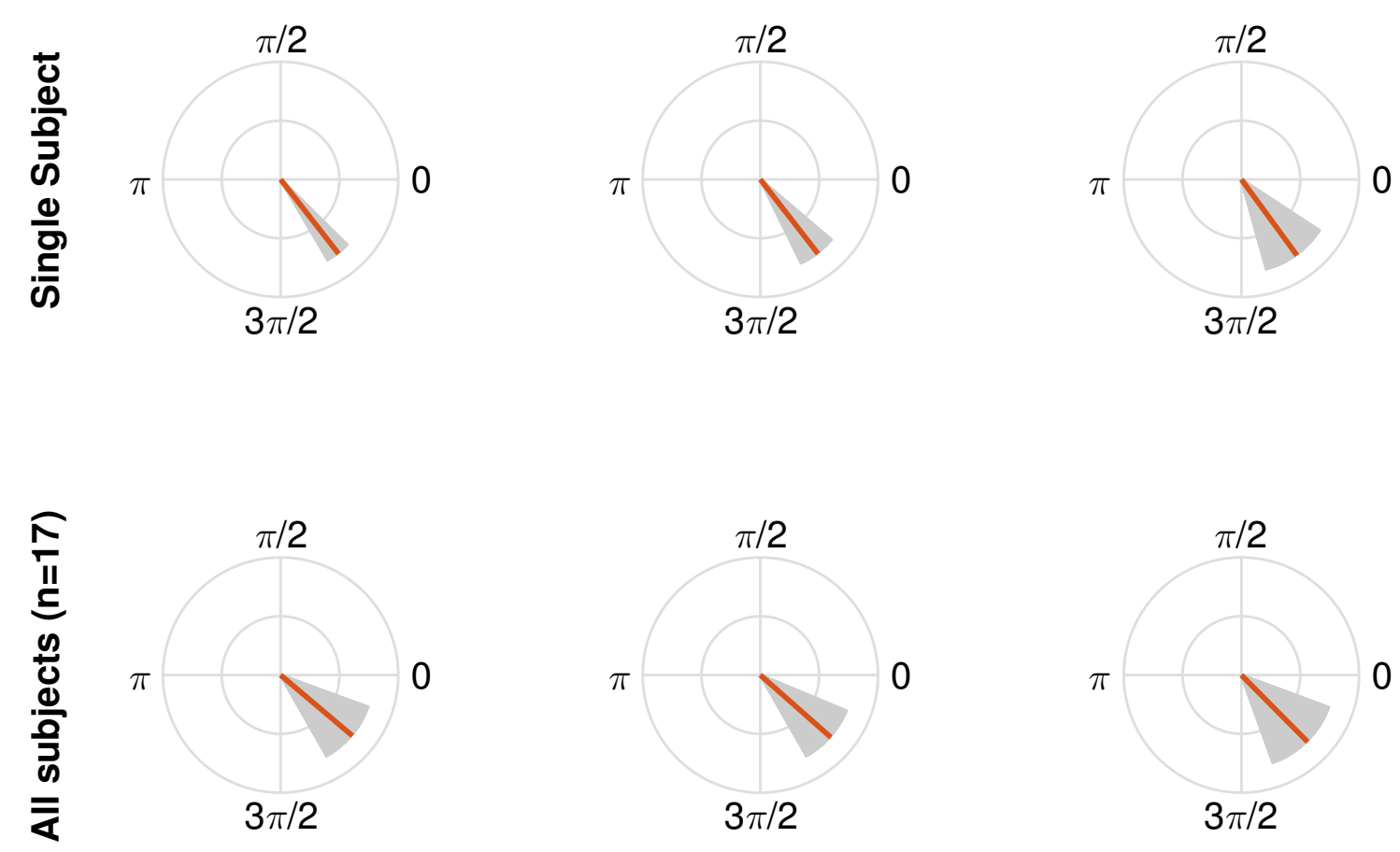

Figure 7: Top: Example circular mean (red line) and circular standard deviation (grey) of phase difference between nasal temperature (NT) and RR intervals for the three techniques in one individual. Bottom: Group circular mean (red) and standard deviation (grey) of circular means ( $\mathrm{n}=17$ ) of phase difference between NT and RR intervals. All pairs of distributions were not statistically different $(p>0.54)$. 

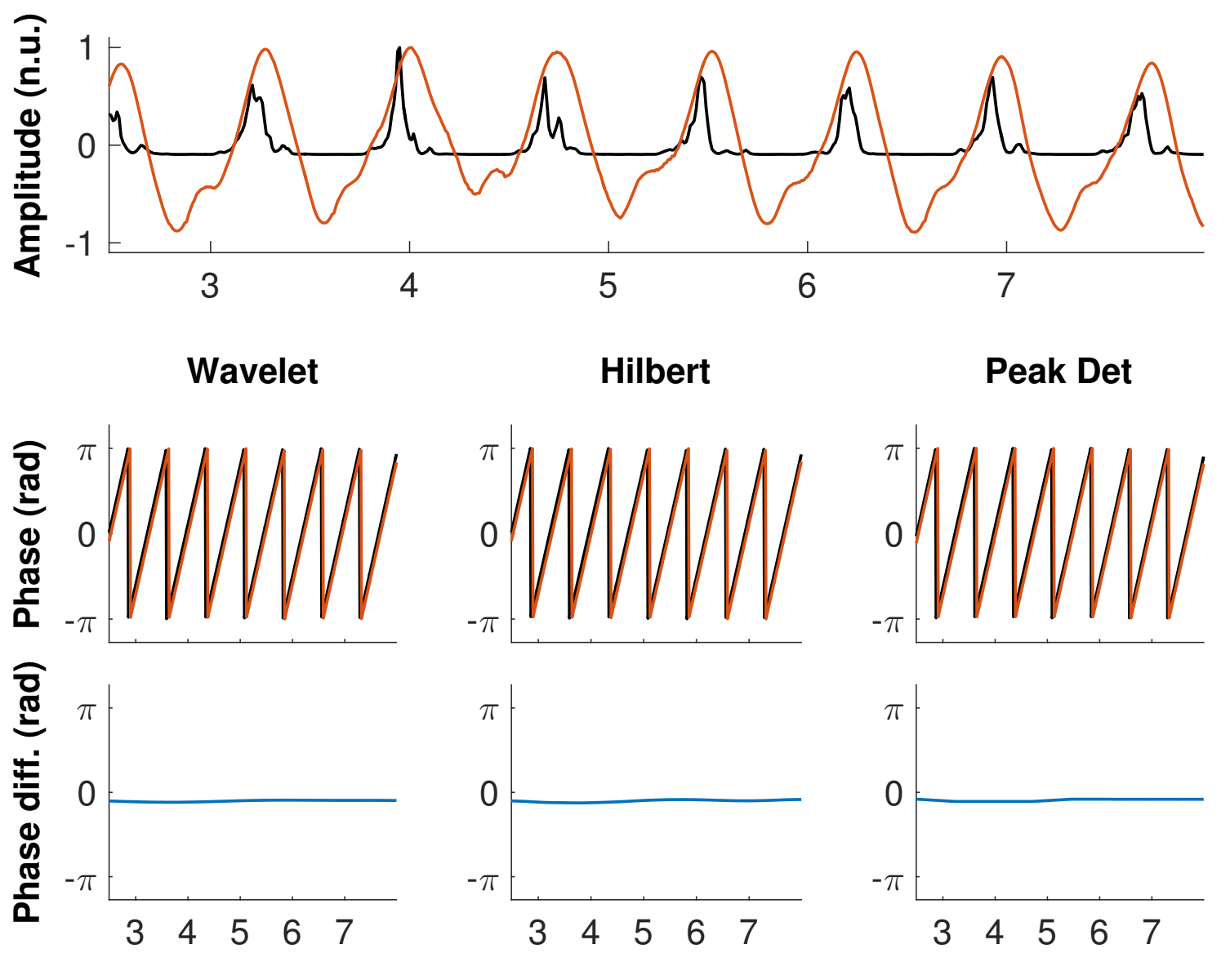

Time (s)

Figure 8: Top: Medial gastrocnemius electromyogram (EMG, black) intensity and medial gastrocnemius muscle-tendon unit (MTU; red) length in one human subject during bicycling at a fixed crank speed of 80 revolutions per minute. Both EMG intensity and MTU length oscillate with the frequency of pedalling. Normalised units were obtained by subtracting the mean and dividing by the resulting maximum amplitude. Middle: The phase of MTU length and EMG intensity are shown as calculated by each technique. Bottom: Phase difference between the two time series. 
Wavelet
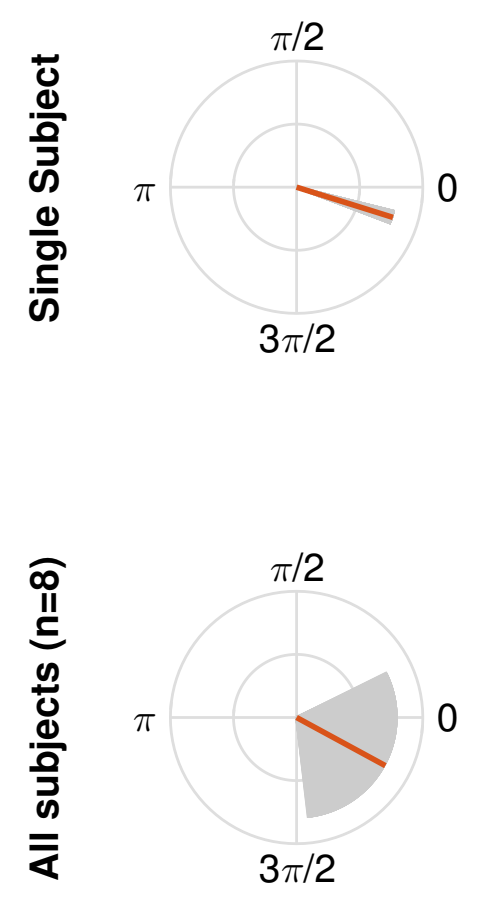

Hilbert
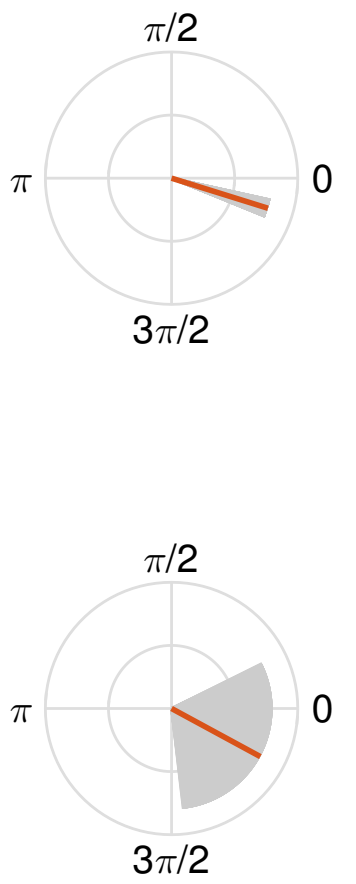

Peak Det
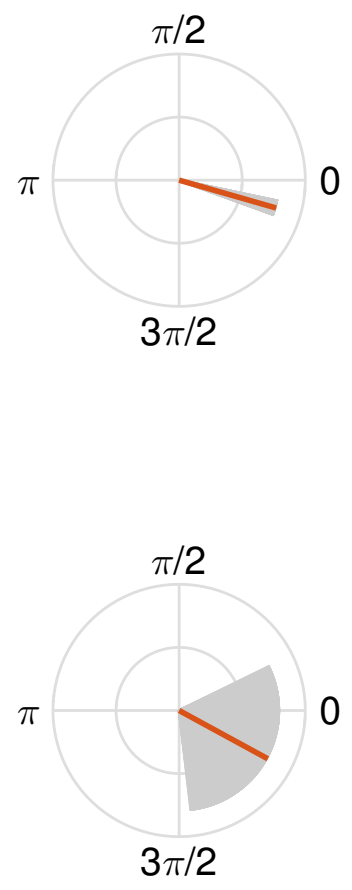

Figure 9: Top: Example circular mean (red line) and circular standard deviation (grey) of phase difference between human EMG intensity and muscle-tendon unit length for the three techniques in one individual. Bottom: Group circular mean (red) and standard deviation (grey) of circular means $(n=8)$ of phase difference between human EMG intensity and muscle-tendon unit length. All pairs of distributions were not statistically different $(p>0.99)$. 

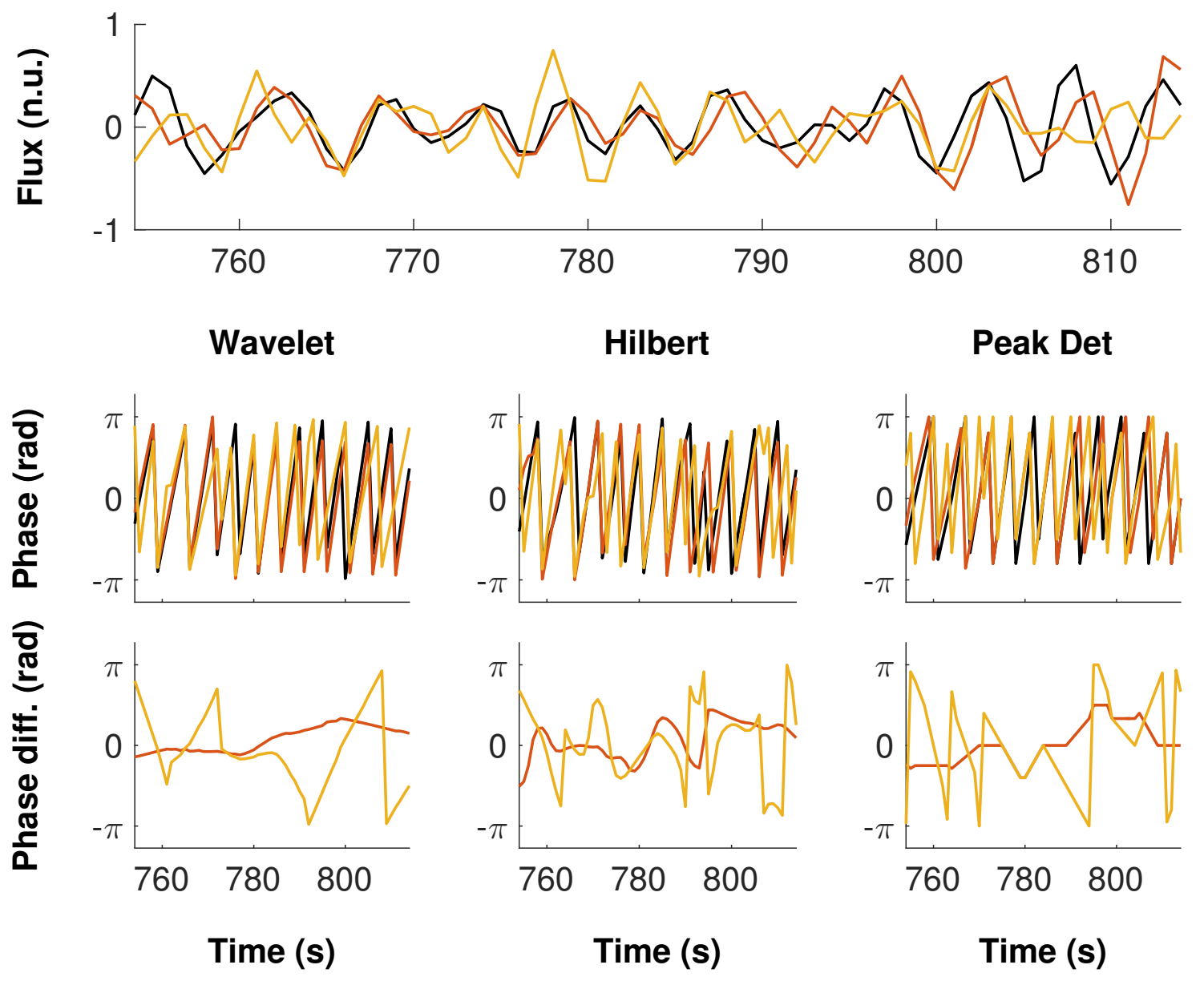

Figure 10: Top: Example filtered renal surface perfusion from three separate regions of interest (ROI). Time series perfusion data are represented as flux, which is an index of blood flow. Two ROI (black, red) are $140 \mu \mathrm{m}$ apart and the third (yellow) is $1.2 \mathrm{~mm}$ away from them. Oscillations around $0.2 \mathrm{~Hz}$ in all three signals are caused by the myogenic response to pressure. Normalised units were obtained by subtracting the mean and dividing by the resulting maximum amplitude. Middle: The phase of each ROI are shown as calculated by each technique.. Bottom: Phase difference between nearby (red: ROI1 vs ROI2) and distant (yellow: ROI1 vs ROI3). 

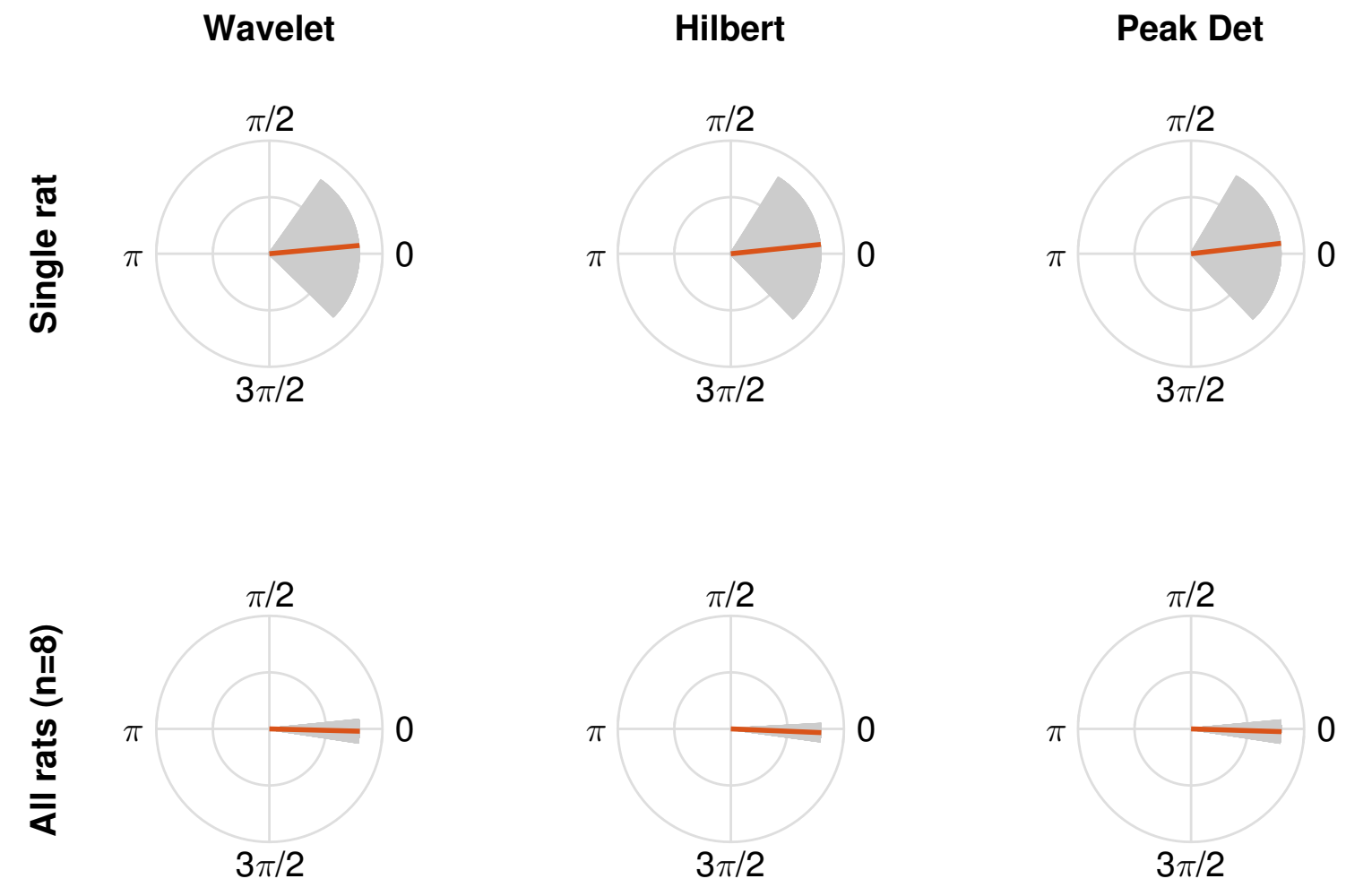

Figure 11: Top: Example circular mean (red line) and circular standard deviation (grey) of the phase difference between two regions of interest that are close to each other for the three techniques in one rat. Bottom: Group circular mean (red) and standard deviation (grey) of circular means $(n=4)$ of phase difference between two regions of interest that are close to each other. All pairs of distributions were not statistically different $(p>0.80)$. 

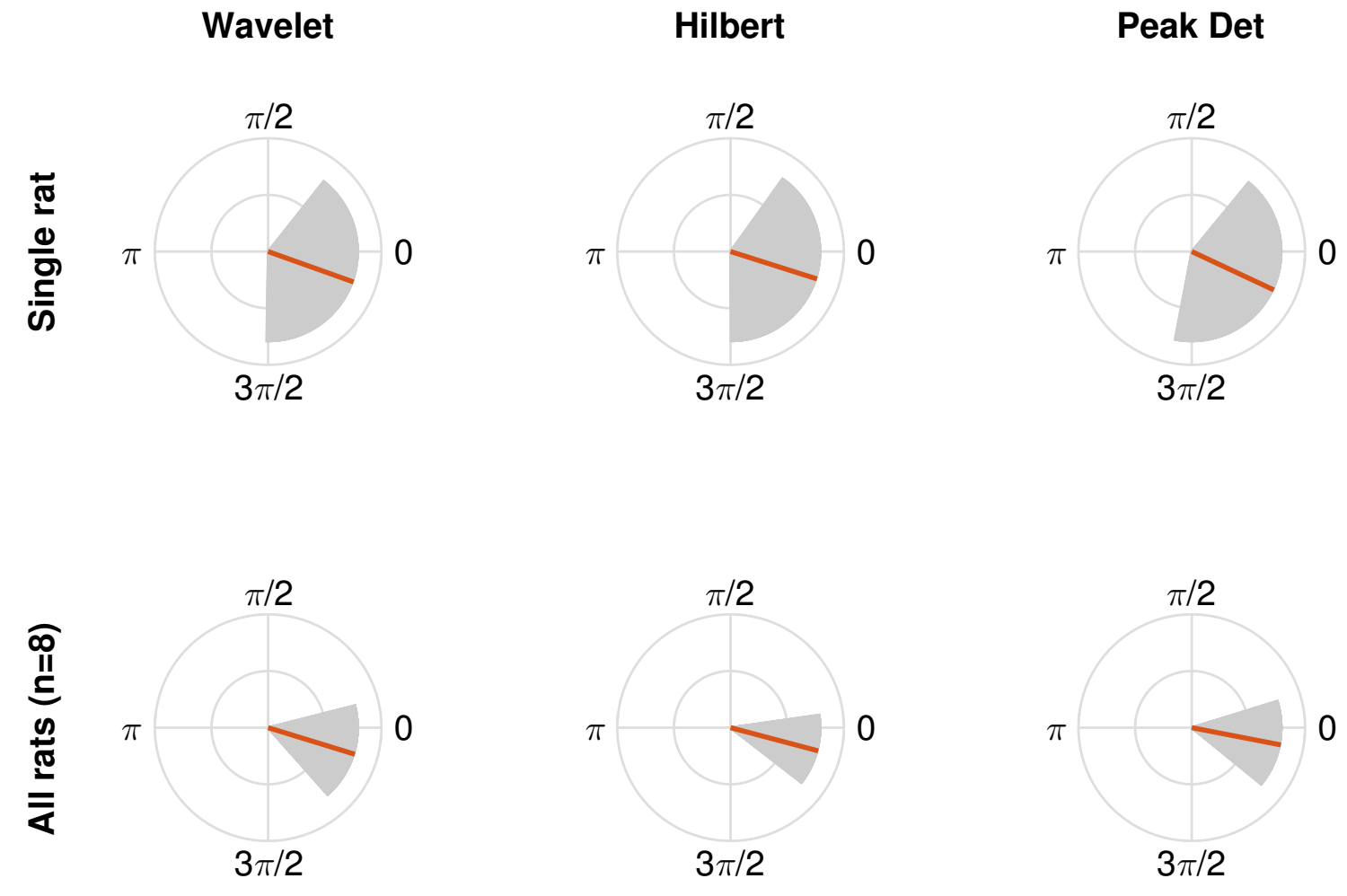

Figure 12: Top: Example circular mean (red line) and circular standard deviation (grey) of the phase difference between two regions of interest that are far from each other for the three techniques in one rat. Bottom: Group circular mean (red) and standard deviation (grey) of circular means $(n=4)$ of phase difference between two regions of interest that are far from each other. All pairs of distributions were not statistically different $(p>0.72)$. 